\title{
Effect of Extracellular Calcium Concentration on Controlling Cardiac Alternans
}

\author{
Shiuan-Ni Liang ${ }^{1}$, Pik-Yin Lai ${ }^{2}$ \\ ${ }^{1}$ Electrical and Computer Systems Engineering, School of Engineering, Monash University, Bandar \\ Sunway, Malaysia \\ ${ }^{2}$ Department of Physics, Graduate Institute of Biophysics, and Center for Complex Systems, National \\ Central University, Chungli District, TaoYuan City, Taiwan R.O.C.
}

\begin{abstract}
Cardiac alternans may lead to life-threatening arrhythmias or sudden cardiac death. Thus, finding an effective method to suppress alternans is crucial. In this paper, we demonstrate that the time needed to control cardiac alternans induced by rapid pacing using the $T+T$ feedback control can be dramatically shortened if the extracellular calcium concentration is lowered by a very small amount when the control is turned on. Numerical simulations are performed on the Hund-Rudy dynamic (HRd) model for single cell. Since controlling alternans is important as it can be a precursor of sudden cardiac death, the results of our research may have important clinical implications and will lead to the development of a better control scheme for alternans or a smart defibrillator.
\end{abstract}

\section{Introduction}

Cardiac arrhythmia is a condition where heartbeats are abnormal. Every year, millions of people worldwide experience arrhythmias and many of them can return to their normal lives if the arrhythmias are treated at early stages and properly. However, many arrhythmias are dangerous and may lead to sudden cardiac death.

Cardiac alternans $[1,2]$, which can be induced by fast pacing, is the condition of having alternate-beat oscillation in the electrical activity (the action potential durations, APDs, oscillate in a long-short-long-short pattern) or alternating strong and weak beats in a heart under a constant pacing. It may lead to life-threatening cardiac arrhythmias [3,4], such as ventricular tachycardia and ventricular fibrillation, or sudden cardiac death $[4,5]$ if it is left without any further medical treatment or intervention. Therefore, searching for an effective alternans control method is crucial.

Besides the earlier proposed methods for alternans suppression: the proportional feedback control $[6,7]$ and adaptive scheme [8-10], a new control scheme, the $T+T$ feedback control [11-14] was proposed recently. The basic pacing period $T$ in this method is designed to change between two values, $T+\varepsilon$ and $T-\varepsilon$ (where $\varepsilon$ is a predetermined control parameter and is much less than $T$ ), repeatedly until the alternans is suppressed. Experiments $[11,13]$ and theoretical [11-14] studies have been carried out and the results showed that the $T+T$ - control method can suppress alternans effectively. However, when the pacing rate continues to increase, the amplitude of the alternans also increases and becomes more difficult to be controlled. Thus, it is necessary to look into how the $T+T$ control method can be improved to suppress alternans induced by rapid pacing.

During cardiac alternans, the intracellular calcium concentration $\left([\mathrm{Ca}]_{\mathrm{i}}\right)$ can also alternate between high and low levels [15]. Experimental [16] and theoretical [2] studies have shown that suppression of $[\mathrm{Ca}]_{i}$ alternans can eliminate APD alternans. Since $[\mathrm{Ca}]_{\mathrm{i}}$ can be regulated by extracellular calcium concentration ([Ca $\left.]_{\mathrm{o}}\right)$ [17] and there are experimental studies $[18,19]$ showing that lowering extracellular calcium concentration $\left([\mathrm{Ca}]_{0}\right)$ can suppress alternans, it is interesting to examine the combination of $T+T$ - control method and low $[\mathrm{Ca}]_{\mathrm{o}}$ as a possible more effective alternans control method. In this study, we investigated the time needed to suppress cardiac alternans using the $T+T$ - control method with lower $[\mathrm{Ca}]_{\mathrm{o}}$ during the control. The details of the control scheme and numerical calculation are given in the next section, followed by the results. In the last section, we discuss our significant findings and the clinical implications.

\section{Methods}

The Hund-Rudy dynamic (HRd) model of canine ventricular action potential [2,20], which is one of the typical mathematical models used to study cardiac alternans, was used in this study to simulate the alternans of a single cell. The HRd model includes the ionic-currents, pumps, exchangers, dynamic concentration change of ions, and calcium cycling. The rate of change of the membrane potential $(V)$ is described by the following equation: 


$$
\mathrm{d} V / \mathrm{d} t=-\left(I_{\text {ion }}+I_{\text {stim }}\right) / C_{\mathrm{m}}
$$

where $t$ is the time, $C_{\mathrm{m}}$ is the cell membrane capacitance, $I_{\text {ion }}$ is the total membrane current density and $I_{\text {stim }}$ is the stimulus current. During the simulations, the cell was paced with a current stimulus of $-80 \mu \mathrm{A} / \mu \mathrm{F}$ for $1 \mathrm{~ms}$. The numerical integration of the differential equations was performed using the MATLAB subroutine ode15s [21,22], which is a variable-step and variable-order solver, with error tolerance of $10^{-6}$.

In the $T+T$ - control method [11-14] that was employed in our study to suppress the alternans, the basic cycle length at the $n$-th beat $\left(T_{n}\right)$ depends on two successive peak-[Ca $]_{i}$ as follows

$$
\begin{aligned}
T_{n} & =T+\varepsilon \quad \text { if }[\mathrm{Ca}]_{\mathrm{i}, n}>[\mathrm{Ca}]_{\mathrm{i}, n-1} \\
& =T-\varepsilon \quad \text { if }[\mathrm{Ca}]_{\mathrm{i}, n}<[\mathrm{Ca}]_{\mathrm{i}, n-1}
\end{aligned}
$$

where $T$ is the basic cycle length, $\varepsilon$ is a pre-determined parameter and $[\mathrm{Ca}]_{\mathrm{i}, n}$ is the peak-[Ca $]_{\mathrm{i}}$ at the $n$-th beat.

In the simulations, first, the alternans is generated using $[\mathrm{Ca}]_{\mathrm{o}}=1.8 \mathrm{mM}$ (the original value used in the HRd model) for a particular basic cycle length $T$. The alternans has reached a steady state when the peak-[Ca $]_{i}$ alternans showed $<0.1 \%$ variability over 100 alternans (i.e. 200 beats). Then, the control is turned on and, at the same time, the $[\mathrm{Ca}]_{\mathrm{o}}$ is changed to a lower or higher (than $1.8 \mathrm{mM}$ ) value until the alternans is successfully suppressed at beat number $n$, that is, when the criteria

$$
\mathrm{APD}_{n}-\mathrm{APD}_{n+1}<\varepsilon
$$

where $\mathrm{APD}_{n}$ is the APD at the $n$-th beat, is satisfied. The APD is measured from the time at the maximum value of $\mathrm{d} V / \mathrm{d} t$ during depolarization to the time at $90 \%$ repolarization from the maximum value of $V$. Finally, the time needed to suppress the peak-[Ca $]_{i}$ alternans $(\tau)$ is calculated from the beat number when the control is turned on until the first beat number $n$ when the criteria (3) is satisfied.

\section{Results}

To illustrate the general results of our findings, we will use an example based on $T=240 \mathrm{~ms}$ for this purpose. Since the results for APD alternans suppression are similar to that of peak- $[\mathrm{Ca}]_{\mathrm{i}}$ alternans suppression, we will only present the results for controlling peak-[Ca $]_{\mathrm{i}}$ alternans. Figure 1 shows that when $[\mathrm{Ca}]_{\mathrm{o}}=1.8 \mathrm{mM}$ (as in the original HRd model), the peak-[Ca $]_{i}$ alternans exists and the amplitude is about $0.00065 \mathrm{mM}$. When the $[\mathrm{Ca}]_{\circ}$ is decreased (increased), the amplitude of the alternans also decreases (increases). If $[\mathrm{Ca}]_{\mathrm{o}}$ is reduced to $1.5 \mathrm{mM}$ or even lower, then there is no alternans. This implies that lowering $[\mathrm{Ca}]_{\mathrm{o}}$ can suppress alternans and elevating $[\mathrm{Ca}]_{\mathrm{o}}$ will enhance

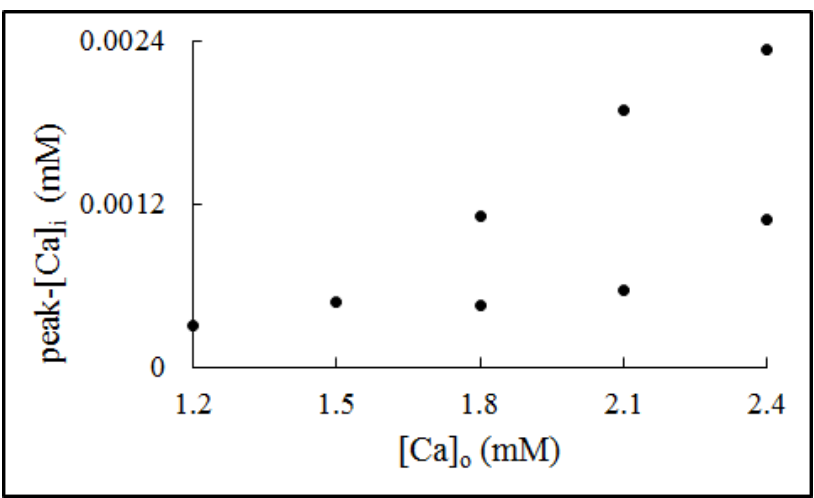

Figure 1. Average peak-[Ca $]_{i}$ of 100 beats (for no alternans cases) or alternans (for alternans cases, that is, over 200 beats) for different $[\mathrm{Ca}]_{\mathrm{o}}$ when $T=240 \mathrm{~ms}$. $[\mathrm{Ca}]_{\mathrm{o}}=1.8 \mathrm{mM}$ is the value used in the original HRd model.

alternans.

Figures 2 and 3 show the control of alternans using the $T+T$ - control scheme with $\varepsilon=5.7 \mathrm{~ms}$, which is very near the critical value of $5.68 \mathrm{~ms}$, when $T=240 \mathrm{~ms}$ (there is a

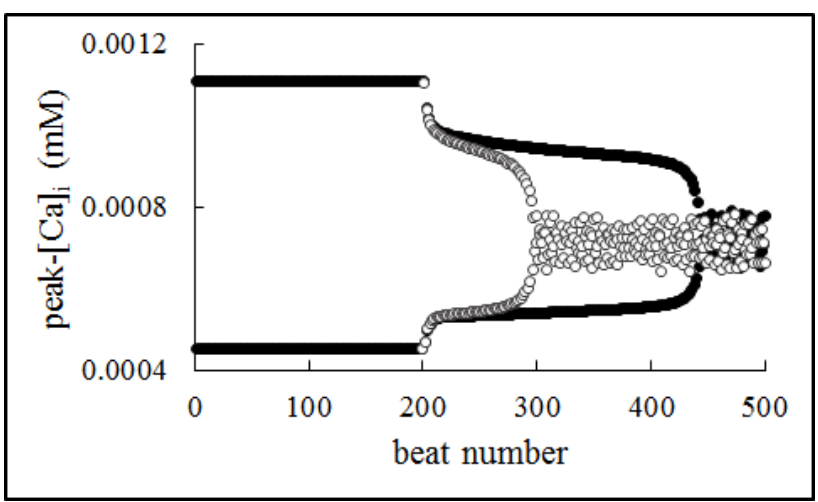

Figure 2. The time sequences of peak-[Ca $]_{i}$ when the control is turned on at beat number 201 with $\varepsilon=5.7 \mathrm{~ms}$ and $T=240 \mathrm{~ms}$ : (filled circles) $[\mathrm{Ca}]_{\mathrm{o}}=1.8 \mathrm{mM}$ and (unfilled circles) $[\mathrm{Ca}]_{0}=1.79 \mathrm{mM}$ when the control is turned on.

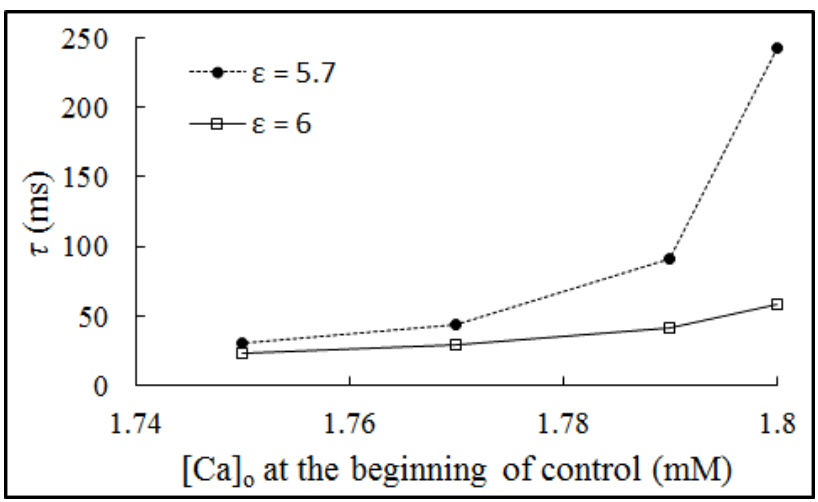

Figure 3. The time needed to suppress the alternans $(\tau)$ for different $[\mathrm{Ca}]_{\mathrm{o}}$ at the beginning of the control with $\varepsilon=5.7$ and $6 \mathrm{~ms}$, and $T=240 \mathrm{~ms}$. 
high possibility that the alternans cannot be suppressed if $\varepsilon$ is lower than its critical value [11-13]). If [Ca] remained at $1.8 \mathrm{mM}$ when the control is turned on, we get $\tau=243$ beats. However, if $[\mathrm{Ca}]_{\mathrm{o}}$ is decreased by $0.01 \mathrm{mM}$ $(0.6 \%)$ to $1.79 \mathrm{mM}$ when the control is turned on, we have $\tau=91$ beats, which is decreased by $62.5 \%$. If the $[\mathrm{Ca}]_{\mathrm{o}}$ is further decreased to $1.75 \mathrm{mM}$ (decreased by $2.8 \%$ from 1.8 $\mathrm{mM}$ ) at the beginning of the control, it is found that $\tau$ can be further reduced to just 30 beats (decreased by $87.7 \%$ compared to $\tau=243$ ). This implies that the control time needed to suppress the alternans can be dramatically reduced by lowering a small percentage of $[\mathrm{Ca}]_{0}$ when the $T+T$ - control is turned on with $\varepsilon$ near its critical value.

\section{Conclusion}

In summary, we have shown that the effectiveness of alternans suppression by using the $T+T$ - control scheme can be improved by changing the $[\mathrm{Ca}]_{\mathrm{o}}$ during control. In particular, the time needed to suppress the peak-[Ca $]_{i}$ alternans, and thus, the APD alternans, can be dramatically shortened if a very small amount of $[\mathrm{Ca}]_{\mathrm{o}}$ is reduced at the beginning of the control. Since controlling alternans is important as it can be a precursor of sudden cardiac death, the results of our research may have important clinical implications and lead to the development of a better control scheme for alternans or a smart defibrillator.

\section{Acknowledgements}

Part of this work is supported by the Ministry of Science and Technology research grant of Taiwan R.O.C..

\section{References}

[1] Shiferaw Y, Karma A. Turing instability mediated by voltage and calcium diffusion in paced cardiac cells. PNAS 2006 Apr; 103(15): 5670-5.

[2] Livshitz LM, Rudy Y. Regulation of $\mathrm{Ca}^{2+}$ and electrical alternans in cardiac myocytes: role of CAMKII and repolarizing currents. Am. J. Physiol. Heart Circ. Physiol. 2007 Jun; 292(6): H2854-66.

[3] Merchant FM, Sayadi O, Puppala D, Moazzami K, Heller V, Armoundas AA. Am. J. Physiol. Heart Circ. Physiol. A translational approach to probe the proarrhythmic potential of cardiac alternans: a reversible overture to arrhythmogenesis? 2014 Feb; 306(4): H465-74.

[4] Qu Z, Xie Y, Garfinkel A, Weiss JN. T-wave alternans and arrhythmogenesis in cardiac diseases. Front. Physiol. 2010; 1: 154.

[5] Qu Z, Garfinkel A, Chen PS, Weiss JN. Mechanisms of discordant alternans and induction of reentry in simulated cardiac tissue. Circulation 2000; 102: 1664-70.

[6] Hall K, Christini DJ, Tremblay M, Collins JJ, Glass L, Billette J. Dynamic control of cardiac alternans. Phys. Rev. Lett. 1997 Jun; 78(23): 4518-21.

[7] Hall GM, Gauthier DJ. Experimental control of cardiac muscle alternans. Phys. Rev. Lett. 2002 May; 88(19): 198102.

[8] Jordan PN, Christini DJ. Adaptive diastolic interval control of cardiac action potential duration alternans. J. Cardiovasc. Electrophysiol. 2004 Oct; 15(10): 1177-85.

[9] $\mathrm{Qu} \mathrm{Z}$. Nonlinear dynamic control of irregular cardiac rhythms. J. Cardiovasc. Electrophysiol. 2004 Oct; 15(10): 1186-7.

[10] Berger CM, Cain JW, Socolar JES, Gauthier DJ. Control of electrical alternans in simulations of paced myocardium using extended time-delay autosynchronization. Phys. Rev. E 2007 Oct; 76 (4 Pt 1): 041917.

[11] Sridhar S, Le DM, Mi YJ, Sitabhra S, Lai PY, Chan CK. Suppression of cardiac alternans by alternating period feedback stimulations. Phys. Rev. E 2013; 87: 042712.

[12] Liang SN, Le DM, Lai PY, Chan CK. Ionic characteristics in cardiac alternans suppression using $T \pm \varepsilon$ feedback control. Europhys. Lett. 2016; 115: 48001.

[13] Le DM, Lin YT, Yang YT, Lai PY, Chan CK. Cardiac alternans reduction by chaotic attractors in $\mathrm{T} \pm \varepsilon$ feedback control. Europhys. Lett. 2017; 117: 50001.

[14] Liang SN, Lai PY. Suppression of cardiac alternans by the alternating-period feedback control. In: Lee YK, Radzol ARM, editors. 2016 IEEE EMBS Conference on Biomedical Engineering and Sciences (IECBES). Danvers (MA): IEEE, 2016:657-9.

[15] Qu Z, Nivala M, Weiss JN. Calcium alternans in cardiac myocytes: order from disorder. J. Mol. Cell Cardiol. 2013; 58: 100-9.

[16] Kanaporis G, Blatter LA. The mechanisms of calcium cycling and action potential dynamics in cardiac alternans. Circ. Res. 2015 Feb; 116(5): 846-56.

[17] Leitch SP and Brown HF. Effect of raised extracellular calcium on characteristics of the guinea-pig ventricular action potential. J Mol Cell Cardiol 1996; 28: 541-51.

[18] Shimizu W, Antzelevitch C. Cellular and ionic basis for Twave alternans under long-QT conditions. Circulation 1999; 99: 1499-507.

[19] Lab MJ, Seed WA. Pulsus alternans. Cardiovascular Research 1993 Aug; 27(8): 1407-12.

[20] Hund TJ, Rudy Y. Rate dependence and regulation of action potential and calcium transient in a canine cardiac ventricular cell model. Circulation 2004; 110: 3168-74.

[21] Shampine LF, Reichelt MW. The Matlab ODE suite. SIAM J. Sci. Comput. 1997; 18: 1-22.

[22] Shampine LF, Reichelt MW, Kierzenka JA. Solving index-1 DAEs in MATLAB and Simulink. SIAM Rev. 1999; 41: 538-552.

Address for correspondence.

Shiuan-Ni Liang

Electrical and Computer Systems Engineering, School of Engineering, Monash University, 47500 Bandar Sunway, Malaysia.

liang.shiuan-ni@monash.edu 
Page 4 